\title{
Aquaporin 7 involved in GINSENOSIDE- RB1-mediated anti-obesity via peroxisome proliferator-activated receptor gamma pathway
}

Rong Guo ${ }^{1,2,3 \dagger}$, Lei Wang ${ }^{1 \dagger}$, Xianqin Zeng ${ }^{2,3}$, Minghao Liu' ${ }^{1,4}$, Peng Zhou' ${ }^{1}$, Huixia Lu' ${ }^{1}$ Huili Lin ${ }^{2^{*}}$ and Mei Dong ${ }^{1 *}$ (D)

\begin{abstract}
Background: Obesity, characterized by the excessive accumulation of triglycerides in adipocytes and their decreased excretion from adipocytes, is closely related to various health problems. Ginsenoside Rb1 (Rb1), the most active component of the traditional Chinese medicine ginseng, has been reported to have positive effects on lipid metabolism. The aim of the present study was to determine the protective effects of Rb1 on glycolipid metabolism under obesity conditions and its mechanisms and to reveal the signaling pathways involved.

Methods: In our study, male C57BL/6 mice with obesity induced by a high-fat diet (HFD) and mature 3 T3-L1 adipocytes were used to investigate the role of Rb1 in lipid accumulation and explore its possible molecular mechanism in vivo and in vitro, respectively.
\end{abstract}

Results: $\mathrm{Rb} 1$ reduced the body weight, fat mass, adipocytes size and serum free fatty acid (FFA) concentration of obese mice. In differentiated 3 T3-L1 adipocytes, Rb1 reduced the accumulation of lipid droplets and stimulated output of triglycerides. Additionally, the expression of peroxisome proliferator-activated receptor gamma (PPARY), phosphorylated PPARY (Ser112) and aquaporin 7 (AQP7) was upregulated in adipocytes and adipose tissues upon Rb1 treatment. However, intervention of GW9662, PPARY antagonist, attenuated Rb1-mediated effects on glycolipid metabolism and AQP7 levels.

Conclusions: These data indicated that Rb1 reduced body weight and improved glycolipid metabolism by upregulating PPARY and AQP7 protein levels. Our study indicated a potential role for Rb1 in the prevention and treatment of obesity.

Keywords: Ginsenoside Rb1, Obesity, Fat, Aquaporin 7, PPARY, Lipid release

\footnotetext{
*Correspondence: linhuilijinan@aliyun.com; meidong_sdu@163.com

${ }^{\dagger}$ Rong Guo and Lei Wang contributed equally to this work.

2Department of Cardiology, The Second Affiliated Hospital of Fujian Medical

University, Quanzhou 362000, Fujian, People's Republic of China

${ }^{1}$ The Key Laboratory of Cardiovascular Remodeling and Function Research,

Chinese Ministry of Education, Chinese National Health Commission and

Chinese Academy of Medical Sciences, The State and Shandong Province

Joint Key Laboratory of Translational Cardiovascular Medicine, Department of

Cardiology, Qilu Hospital, Cheeloo College of Medicine, Shandong University,

107 Wenhuaxi Road, Jinan 250012, China

Full list of author information is available at the end of the article
}

(C) The Author(s). 2020 Open Access This article is licensed under a Creative Commons Attribution 4.0 International License, which permits use, sharing, adaptation, distribution and reproduction in any medium or format, as long as you give appropriate credit to the original author(s) and the source, provide a link to the Creative Commons licence, and indicate if changes were made. The images or other third party material in this article are included in the article's Creative Commons licence, unless indicated otherwise in a credit line to the material. If material is not included in the article's Creative Commons licence and your intended use is not permitted by statutory regulation or exceeds the permitted use, you will need to obtain permission directly from the copyright holder. To view a copy of this licence, visit http://creativecommons.org/licenses/by/4.0/ The Creative Commons Public Domain Dedication waiver (http://creativecommons.org/publicdomain/zero/1.0/) applies to the data made available in this article, unless otherwise stated in a credit line to the data. 


\section{Background}

Obesity, an increasing global public health issue, serves as a risk factor for an expanding set of metabolic diseases, including cardiovascular disease and type 2 diabetes [1]. The pathophysiological progression of obesity is related to increased lipids, mainly triglycerides, the hyperplastic and hypertrophic growth of adipocytes, and the expansion of adipose tissues especially white adipose tissue [2, 3]. Adipose tissue mass is determined by the storage of triglycerides in adipocytes and their removal from adipocytes. High storage but low removal of triglyceride promotes fat tissue accumulation and obesity [4]. This demonstrates that promoting the rate of triglyceride removal from adipocytes is an important antiobesity therapeutic target.

Aquaporins (AQPs) are transmembrane proteins that facilitate the permeation of water and small solutes across membranes, driven by osmotic of solute gradients [5]. Numerous studies have described the crucial role of aquaglyceroporins, a subfamily of AQPs, in adipose tissue biology and obesity onset $[6,7]$. Under conditions of energy expenditure, triacylglycerol stored in adipocytes is hydrolyzed to glycerol and free fatty acids, which are released into the bloodstream [7]. Among the various AQPs, AQP7 is a vital glycerol transporter in adipocytes [8]. AQP7-depleted mice, showed progressive adipocyte hypertrophy, increased fat mass and metabolic disorders [9]. These effects are thought to be relevant to reduced adipocyte glycerol permeability and the subsequent accumulation of intracellular glycerol and triacylglycerol [10]. The modulation of adipocyte AQP7 expression has been proposed as a target in obesity therapy [11]. The upregulation of AQP7 expression or its functional activation might be an ideal approach for the treatment of obesity. AQP7 is a novel adipose-specific target gene of PPARy [12]. AQP7 expression in adipocytes was reported to be increased by thiazolidinediones, which are synthetic PPAR ligands. PPAR $\gamma$ regulates AQP7 expression through binding of the PPAR $\gamma$-retinoid $\mathrm{X}$ receptor complex to the peroxisome proliferator-activated receptor response element (PPRE) region in the AQP7 gene promoter.

Panax ginseng, a traditional herbal medicine, has been widely used to treat various diseases in Eastern Asia for more than 4000 years. Ginsenoside Rb1 is the most abundant active component of Panax ginseng, has been reported to decrease body weight, ameliorate glycolipid metabolism and reduce triglyceride accumulation [1315]. In addition, PPAR $\gamma$ was reported to be involved in the effects of Rb1 on adipocytes and adipose tissue. Although these studies implied that $\mathrm{Rb} 1$ can reduce fat mass and body weight, its molecular mechanism still remains unclear. Our previous studies also have revealed that $\mathrm{Rb} 1$ reduced lipid accumulation in macrophage foam cells [16], and improved the metabolic environment by inhibiting inflammatory reactions in atherosclerosis models [17].

However, whether Rb1 can regulate AQP7 expression through PPAR $\gamma$ to promote lipid transport and reduce body weight remains unclear.

\section{Methods}

\section{Animal care and treatment}

A total of 90 C57BL/6 mice (male, 4-5 weeks old) were obtained from Charles River Laboratories Animal Technology Company (Beijing, China). All mice were raised on a 12-h light/12-h dark cycle. After one week of adaptation, the mice were randomly divided into two groups and received different diets. Some mice were fed a normal diet $(20.6 \% \mathrm{kcal}$ from protein, $67.4 \% \mathrm{kcal}$ from carbohydrate and $12 \% \mathrm{kcal}$ from fat $)(n=30)$, and the rest were fed a high-fat diet (HFD, 20\% kcal from protein, $20 \% \mathrm{kcal}$ from carbohydrate and 60\% kcal from fat (soybean oil and lard) $(n=60)$ for 12 weeks. Mice fed a normal diet were randomly divided into 2 subgroups $(n=15$ per group): the Chow group (fed a normal diet and treated with saline) and Chow $+\mathrm{Rb} 1$ group (fed a normal diet and treated with Rb1). The body weights were measured weekly. Mice fed a HFD were confirmed as obese if their weight was $20 \%$ greater than that of mice in the Chow group. Subsequently, mice fed a HFD were randomly divided into 4 subgroups ( $n=15$ per group): the HFD group, HFD + Rb1 group, $\mathrm{HFD}+\mathrm{GW} 9662+\mathrm{Rb} 1$ group and HFD + dimethylsulfoxide (DMSO) + Rb1 group. All groups treated with $\mathrm{Rb} 1$ were administered $40 \mathrm{mg} / \mathrm{kg} / \mathrm{d}$ Rb1 (B21050, Shyuanye, China). Mice in the HFD group received an equal volume of saline. DMSO (D8371, Solarbio Beijing, China) was diluted to $1 \%$ in normal saline, and GW9662 (M6191, Sigma US) was prepared in 1\% DMSO before administration. All treatments were conducted by daily intraperitoneal injection for 5 weeks. The injection volume of each liquid was $300 \mu \mathrm{l}$. GW9662 and DMSO treatments were administered $0.5 \mathrm{~h}$ later than $\mathrm{Rb} 1$ treatment, and mice in the HFD + Rb1 group were administered $300 \mu \mathrm{l}$ of normal saline after injection of $300 \mu \mathrm{l}$ of Rb1. After 5 weeks of treatment, the mice were euthanized. Subcutaneous, visceral and epididymal adipose tissues were harvested, and the weight of the fat mass divided by the body weight of mice was considered the body fat rate (BFR).

\section{Glucose tolerance test}

The intraperitoneal glucose tolerance test (IPGTT) was conducted on the 16th week. After fasting overnight, mice were injected intraperitoneally with glucose at a dose of $2 \mathrm{~g} / \mathrm{kg}$ body weight. Blood glucose concentrations in blood from the tail vein of the mice were measured at 0 (fasting), 15, 30, 60 and 120 min after glucose administration. 


\section{Serum biochemical analysis}

Serum collected from each mouse was centrifuged at $3000 \mathrm{rpm}$ for $15 \mathrm{~min}$ and stored at $-80^{\circ} \mathrm{C}$ for further analysis. Blood biochemical tests to detect serum lipids (cholesterol, triglyceride, high density lipoprotein cholesterol, low density lipoprotein cholesterol, and free fatty acid) and blood glucose were performed as described in our previous report [18].

\section{Histological analysis}

Adipose tissue was fixed overnight in $4 \%$ paraformaldehyde, and then embedded in paraffin. Tissue sections $(5 \mu \mathrm{m})$ were collected and mounted onto slides. The sections were stained with hematoxylin and eosin and photographed at $100 \times$ magnification. Using Image-Pro Plus 6.0 software (IPP 6.0, Media Cybernetics, Rockville, MD, United States), at least two fields per slice and six slices per fat mass were analyzed to quantify adipocyte size.

\section{Cell culture and treatment}

Mouse 3 T3-L1 preadipocytes were purchased from American Type Culture Collection (ATCC, US). The cells were cultured in high glucose Dulbecco's modified Eagle medium (DMEM) with 10\% fetal bovine serum (FBS) at $37^{\circ} \mathrm{C}$ in a saturated humidity atmosphere of $5 \%$ $\mathrm{CO}_{2}$. The classic cocktail method was used to induce 3 T3-L1 preadipocyte differentiation. Briefly, two days after reaching contact inhibition, cells were treated with a mixture of $0.5 \mathrm{mM}$ 3-isobutyl-1-methylxanthine, $1 \mu \mathrm{M}$ dexamethasone, and $10 \mu \mathrm{g} / \mathrm{mL}$ insulin in DMEM containing 10\% FBS for 2 days. The media were replaced with DMEM containing $10 \mu \mathrm{g} / \mathrm{mL}$ insulin and $10 \%$ FBS, after which cells were incubated for another 2 days, and the growth media used for an additional day consisted of only DMEM with $10 \%$ FBS. When more than $90 \%$ of the 3 T3-L1 preadipocytes had been induced into mature 3 T3-L1 adipocytes with accumulated lipid droplets in the cytoplasm, they could be used for further study. The optimum dose of Rb1 was $40 \mu \mathrm{M}$, which was determined by titration with different concentrations of Rb1 for 24 h. The dose of GW9662 used was $5 \mathrm{mM}$, and the dose of DMSO used was $0.01 \%$.

\section{Oil red $\mathrm{O}$ staining and triglyceride assay}

Lipid droplets in the cytoplasm were measured using oil red O staining. Differentiated 3 T3-L1 adipocytes were placed on cover slides in plates and treated with the corresponding drug for $24 \mathrm{~h}$. Cells were washed with phosphate-buffered saline two times and fixed with $4 \%$ paraformaldehyde for $2 \mathrm{~h}$ at room temperature. The culture supernatant was collected and used to detect the content of free triglycerides, leptin and adiponectin with an ELISA kit (E1003, Applygen, China). Fixed cells were washed once with distilled water and $60 \%$ isopropanol. Subsequently, $0.5 \%$ oil red $\mathrm{O}$ was added to the cells and incubated for $1 \mathrm{~h}$. The surface dye was rinsed away with distilled water. Cells were observed under a microscope at $100 \times$ magnification.

\section{Western blot analysis}

The total protein was extracted from 3 T3-L1 adipocytes and mouse epididymal adipose tissues with a total protein extraction kit (P1250, Applygen). Protein expression levels of AQP7 and PPAR $\gamma$ were detected. Samples were separated by $10 \%$ SDS-PAGE and transferred onto $0.45 \mu \mathrm{m}$ PVDF membranes (Bio-Rad). The membranes were blocked in $5 \%$ nonfat dry milk followed by incubation with primary antibodies against AQP7 (AB15568, MerckMillipore, Germany), PPARY (ab45036, Abcam, UK), phosphorylated peroxisome proliferator-activated receptor $\gamma$ (pPPAR $\gamma$, Ser112) (abs130911a, Absin, China) and Tubulin- $\alpha$ (AF7010, Affinity, US) overnight at $4{ }^{\circ} \mathrm{C}$. The secondary antibody (ZSGB-BIO, China) was goat antirabbit immunoglobulin $\mathrm{G}$ antibody. After washing, the immunocomplex was incubated with secondary antibody $(1: 10,000)$ for $2 \mathrm{~h}$. Bands were visualized by chemiluminescence (Millipore Corporation, Billerica, MA, USA).

\section{Statistical analysis}

All results are expressed as the means \pm SEMs. Multiple group comparisons were analyzed by ANOVA followed by Tukey's post hoc test, and the significance of differences between the mean values of two groups were analyzed using the unpaired two-tailed Student's $t$-test. Statistical significance was defined as $p<0.05$.

\section{Results}

$\mathrm{Rb} 1$ reduced body weight and fat mass partially in a PPARY-dependent manner

To confirm the effects of Rb1 on body weight in obese mice, we established a mouse model of obesity through HFD feeding. After 12 weeks of HFD feeding, the body weights of the mice were significantly increased compared with those of mice in the Chow group $(p<0.01)$ (Supplement 1) and reached the standard for obesity [19]. The weights of all HFD-fed mice were measured weekly to observe trends over 17 weeks. Then, we investigated the molecular mechanisms of this change. Studies revealed that PPAR $\gamma$ in adipocytes could be activated by Rb1 treatment [20, 21]. To further determine the role of PPARy in Rb1-induced body weight and fat mass loss, mice were intraperitoneally injected with GW9662, an antagonist of PPARy. The results showed that $\mathrm{Rb} 1$ treatment markedly reduced the body weights of obese mice (Fig. 1a-b) and decreased fat mass accumulation (Fig. 1c). BFR (body fat rate = weight of fat mass/body weight"100\%) was significantly reduced in the Rb1-treated group (Fig. 1d). H\&E staining showed that the in vivo 


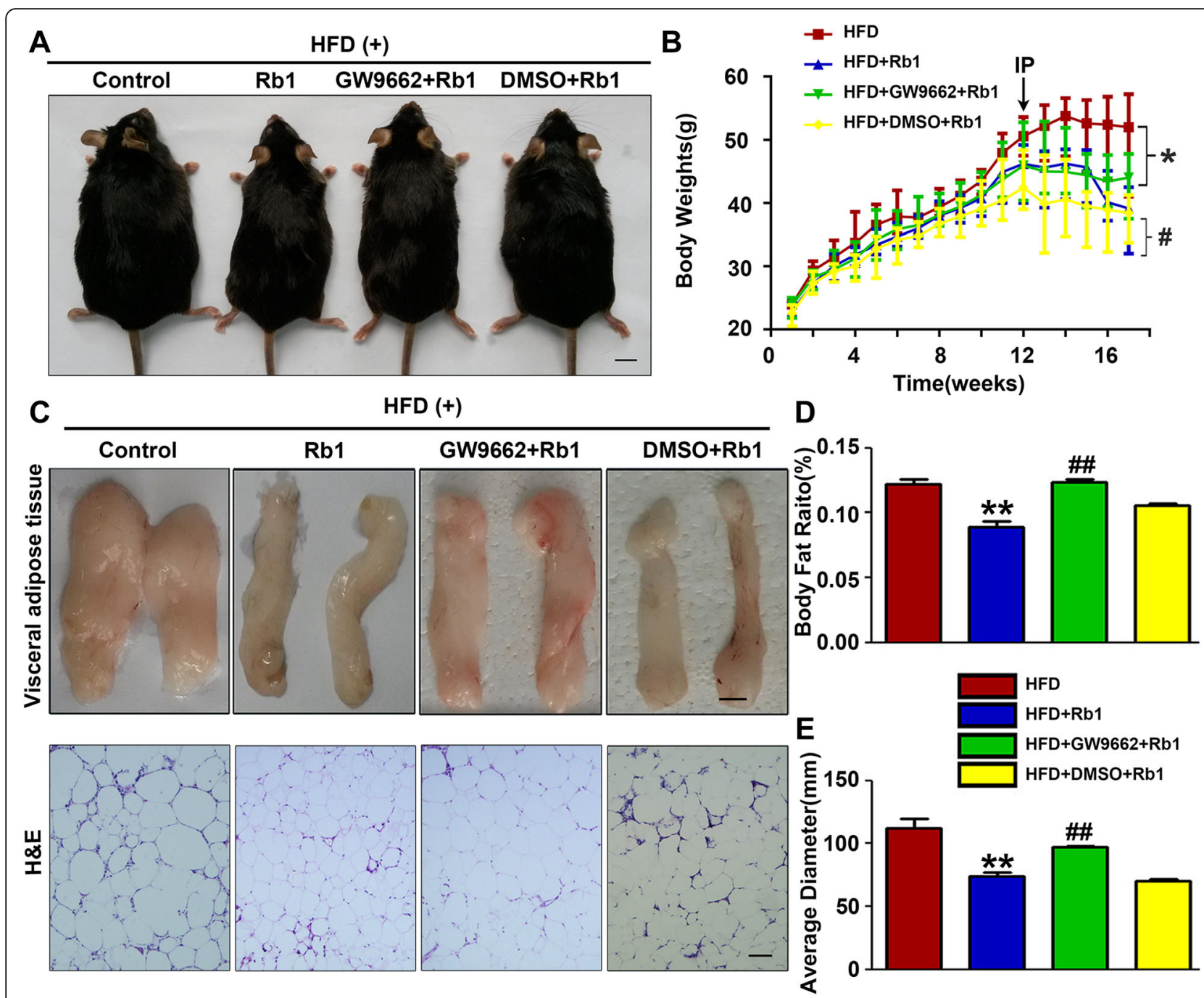

Fig. 1 Effects of Rb1 and/or GW9662 on body weight and fat mass in HFD-induced obese mice. a Representative individuals from the different groups. Scale bar: $1 \mathrm{~cm}$. b Variations in the body weight of the four groups over weeks. $\mathbf{c}$ En face view of visceral adipose tissue from representative individuals from the four groups. Scale bar: $5 \mathrm{~mm}$. H\&E staining of visceral fat tissues. Scale bar: $50 \mu \mathrm{m}$. H\&E staining of visceral fat tissues. Scale bar: $50 \mu \mathrm{m}$. d Quantification of the body fat rate. e Quantification of the average adipocyte diameter. IP: intraperitoneal injection. ${ }^{*} p<0.05$, the HFD + Rb1 group compared with the HFD group; ${ }^{*} p<0.05$, the HFD + GW9662 + Rb1 group compared with the HFD + Rb1 group; ${ }^{* *} p<0.01$, the HFD + Rb1 group compared with the HFD group; ${ }^{* \#} p<0.01$, the HFD + GW9662 + Rb1 group compared with the HFD + Rb1 group

adipocyte size in the HFD + Rb1 group mice was markedly reduced compared with that in the control group (Fig. 1c-e). However, GW9662 treatment partially reversed the decrease in body weight, fat mass and adipocyte size induced by Rb1. There was no significant difference in these parameters in the presence and absence of DMSO, the PPARy dissolvent. These data suggested that the PPARy pathway is involved in the effects of Rb1 in reducing body weight and fat mass in HFD-induced obese mice.

$\mathrm{Rb} 1$ improved the glucose tolerance and influenced the serum lipid profile of obese mice

As glycolipid metabolism plays a vital role in body weight growth, we investigated whether $\mathrm{Rb} 1$ affects glycolipid metabolism. Serum concertrations of cholesterol (TC), triglycerides (TGs), high-density lipoprotein cholesterol (HDL-C), low-density lipoprotein cholesterol (LDL-C), FFAs and blood glucose were detected in HFD-fed mice. The IPGTT was carried out on the 16th week. The area under the curve (AUC) of the IPGTT in the $\mathrm{Rb} 1$ group was reduced compared to that in the HFD group. The AUC of the IPGTT was significantly higher in the GW9662+Rb1 group than in the Rb1 group, and there was no difference in the AUC of the IPGTT between the HFD + DMSO+Rb1 and HFD + Rb1 groups (Fig. 2a-b). Our results showed that Rb1 contributed to the reduced serum FFA levels (Fig. 2c). Serum TG levels tended to decrease with $\mathrm{Rb} 1$ treatment, 


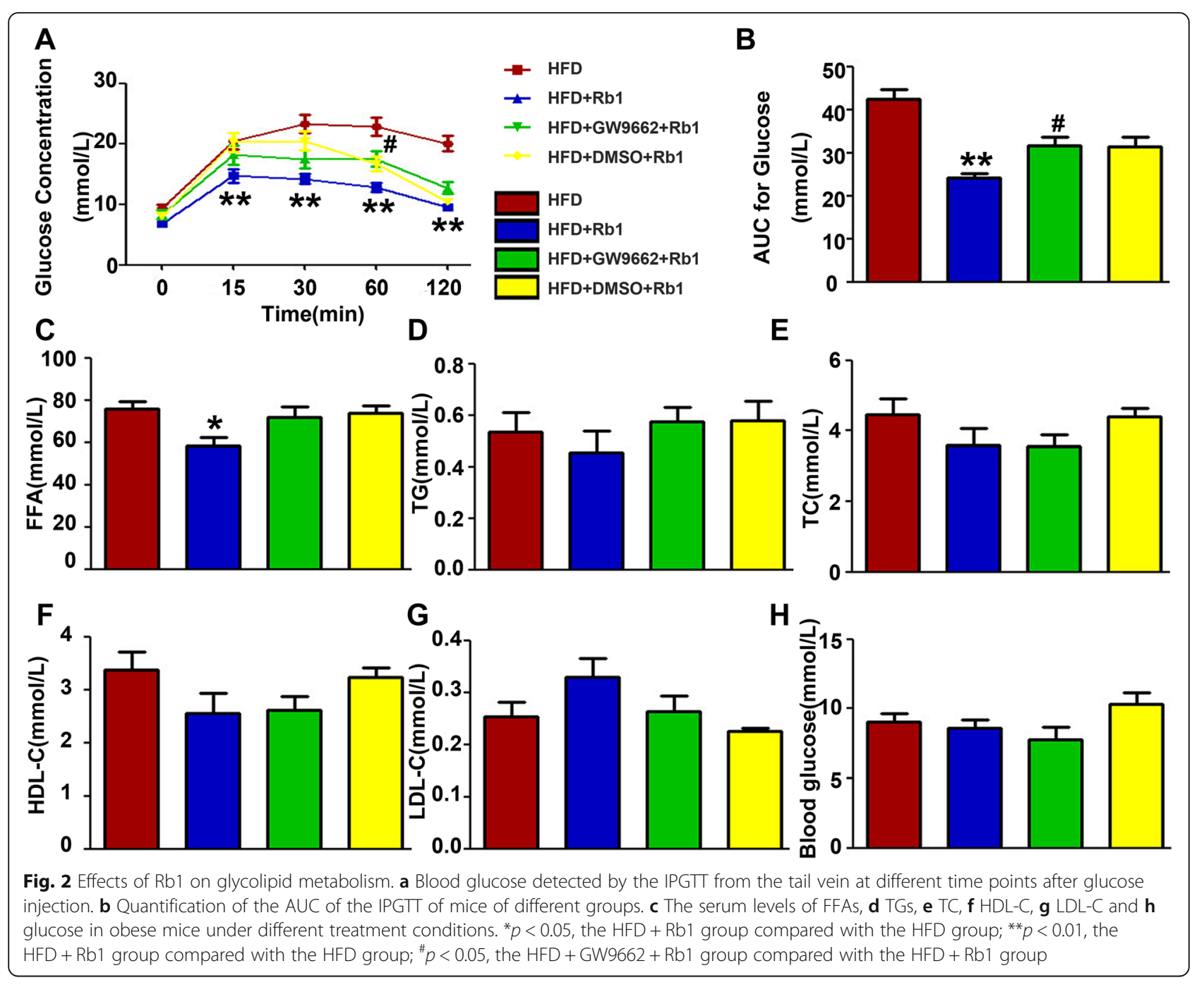

although this difference did not achieve statistical significance. This effect was abrogated by GW9662(Fig. 2d). Serum TC, HDL-C, LDL-C and blood glucose levels were not affected by Rb1 treatment (Fig. 2e-h). In view of these results, Rb1 efficiently lowered the AUC of the IPGTT and improved the ability of obese mice to regulate blood glucose and TG. Inhibition of PPAR $\gamma$ with its antagonist partly reversed this impact. Rb1 decreased the serum level of FFAs, but there was no evidence that PPAR $\gamma$ was involved in the mechanism.

\section{$\mathrm{Rb} 1$ augmented $A Q P 7$ expression in adipose tissue via the PPARY Signaling pathway}

AQP7 is a membrane channel that tranports glycerol and interferes with triglyceride accumulation in adipocytes [22]. It is a target gene of PPAR $\gamma$ and can be activated by PPAR $\gamma$ agonists $[12,23]$. To investigate the effect of Rb1 on AQP7 expression and evaluate the involvement of PPAR $\gamma$, we collected visceral adipose tissue and extracted proteins from the tissue. Rb1 promoted the expression of AQP7, but this effect was partially reversed by a PPARY inhibitor in vivo (Fig. 3a). Meanwhile, Rb1 also upregulated the expression of PPARy (Fig. 3b) and pPPAR (Fig. 3c), and these effects were inhibited by GW9662. The ratio between PPAR $\gamma$ and pPPAR $\gamma$ expression did not change significantly with different treatments (Fig. 3d). These results indicated that Rb1 increased AQP7 expression in a PPAR $\gamma$-dependent manner, which was possibly responsible for its function in inhibiting adipose tissue expansion and reducing body weight. The improvement of glycolipid metabolism with $\mathrm{Rb} 1$ treatment might be associated with the upregulation of PPARy and AQP7.

$\mathrm{Rb} 1$ induced AQP7 and PPARy expression in mature $3 \mathrm{~T} 3-$ L1 adipocytes

To explore the role of $\mathrm{Rb} 1$ in lipid metabolism in vitro, the expression of AQP7, PPARy and pPPAR $\gamma$ was detected in mature $3 \mathrm{~T} 3-\mathrm{L} 1$ adipocytes with or without Rb1 treatment. Differentiated 3 T3-L1 adipocytes were 


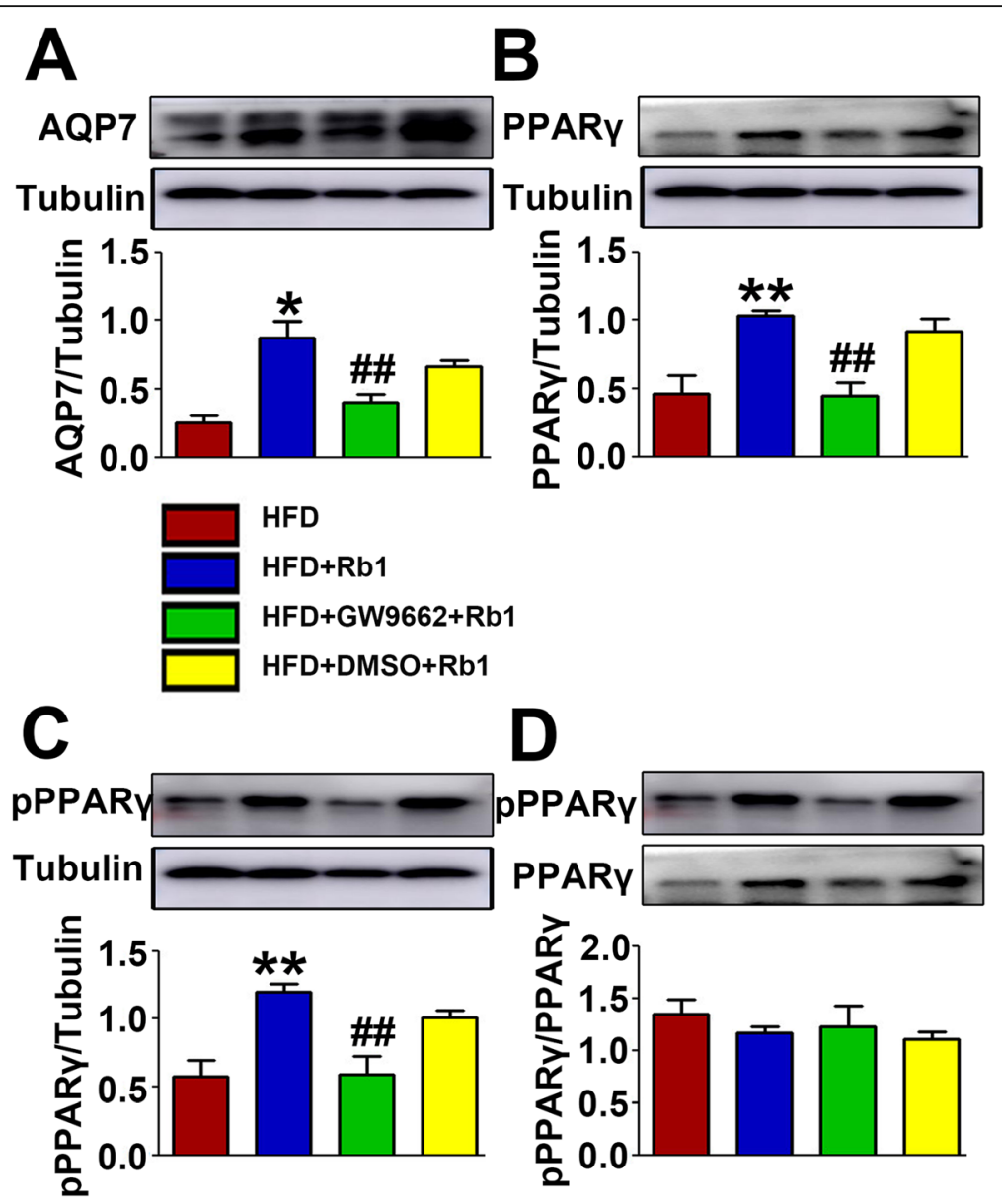

Fig. 3 Rb1 increased the expression levels of AQP7 and PPARy in adipose tissue. a Representative western blot analysis and quantification of AQP7. b Representative western blot analysis and quantification of PPARy. c Representative western blot analysis and quantification of pPPARY. d Representative western blot analysis and quantification of pPPARy/PPARY in vivo. ${ }^{*} p<0.05$, the HFD + Rb1 group compared with the HFD group; ${ }^{* *} p<0.01$, the HFD + Rb1 group compared with the HFD group; ${ }^{\# \#} p<0.01$, the HFD + GW9662 + Rb1 group compared with the HFD + Rb1 group

treated with $\mathrm{Rb} 1$ at different concentrations for $24 \mathrm{~h}$. Our results showed that AQP7 protein levels were upregulated under treatment with 20 to $80 \mu \mathrm{M}$ Rb1 (Fig. 4a-b). The PPAR $\gamma$ protein level was increased with $40 \mu \mathrm{M}$ Rb1 treatment (Fig. 4c) and pPPAR level was increased with $40 \mu \mathrm{M}$ and $80 \mu \mathrm{M}$ Rb1 treatment (Fig. $4 \mathrm{~d}$ ) compared to that in the control group $(0 \mu \mathrm{M} R \mathrm{Rb})$. However, the pPPAR $\gamma /$ PPAR $\gamma$ level was unchanged with Rb1 treatment (Fig. 4e). We selected $40 \mu \mathrm{M} \mathrm{Rb1}$ for use in the following experiments.

Rb1 decreased lipid storage in adipocytes by promoting PPARY/ AQP7 protein expression in mature 3 T3-L1 adipocytes

To further understand the molecular mechanism by which Rb1 regulates AQP7, we inhibited PPARy by incubation with GW9662 in vitro. Oil red O staining showed that the intracellular lipid droplet size was significantly decreased in the Rb1 group (Fig. 5a). The triglyceride concentration in the cell medium was increased with Rb1 treatment (Fig. 5b). Compared to the Rb1 group, the GW9662 + Rb1 group showed larger adipocytes lipids and a lower triglyceride content in the medium. As an endocrine organ, adipose tissue secretes many adipocytokines [24]. Leptin and adiponectin, typical adipokines, play important roles in lipodystrophy. The expression of adiponectin decreases with an increase in adiposity, while leptin levels increase in obesity [25]. To obtain a deeper understanding of the effects of $\mathrm{Rb} 1$, we measured the concentrations of leptin and adiponectin in adipocytes. Leptin levels in the culture supernatant were decreased by Rb1 treatment (Fig. 5c). $\mathrm{Rb} 1$ increased adiponectin levels in the cell culture medium of adipocytes (Fig. 5d). These effects of Rb1 on leptin and adiponectin could be inhibited by GW9662. These results showed that Rb1 could influence the metabolic state of adipocytes through the PPARY pathway.

Our results indicated that Rb1 augmented AQP7 protein expression in adipocytes, which was suppressed by a PPAR $\gamma$ antagonist (Fig. 5e-f). This result was consistent 


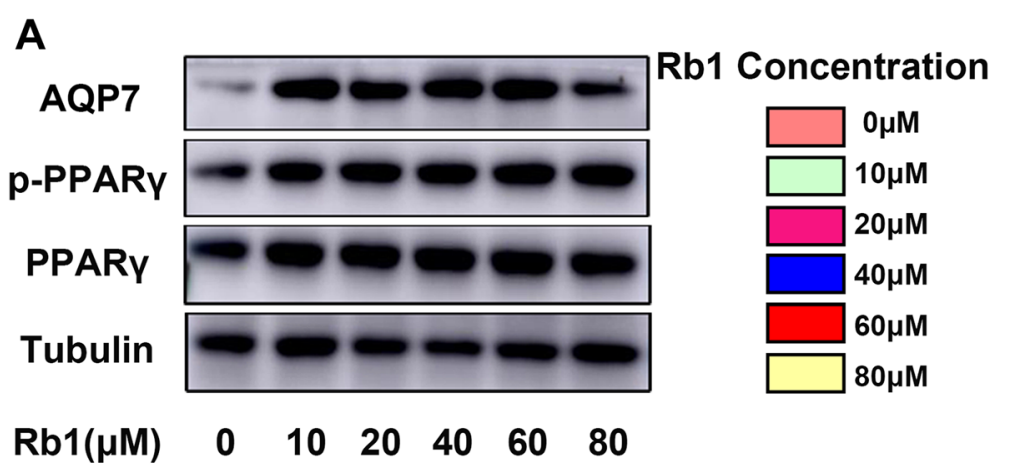

B

C

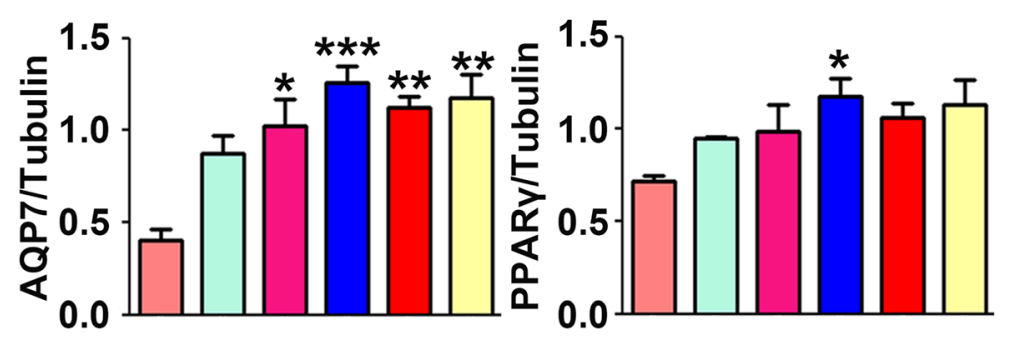

D

E

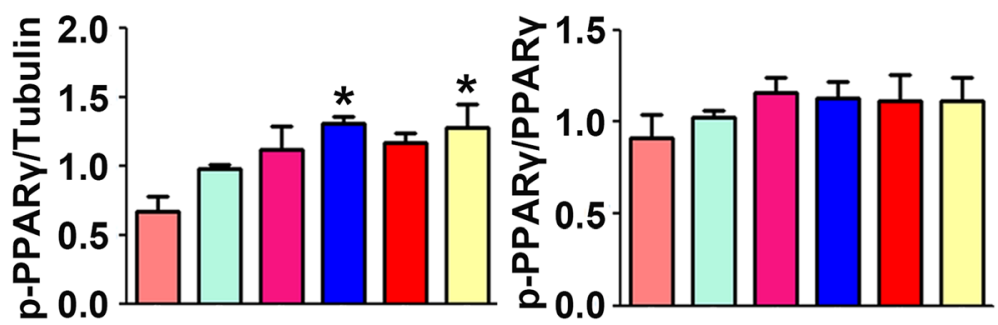

Fig. 4 AQP7, PPPARY and PPARy expression in mature 3 T3-L1 adipocytes treated with Rb1 at different concentrations. a Representative western blot analysis of AQP7, PPPARY and PPARY expression in mature 3 T3-L1 adipocytes treated with Rb1 at different concentrations. b Quantification of AQP7, c PPARY, $\mathbf{d}$ PPPARY and e pPPARy/PPARy expression relative to the tubulin level in different groups. ${ }^{*} p<0.05$, compared with the control group $(0 \mu \mathrm{M} \mathrm{Rb} 1)$; ${ }^{* *} p<0.01$, compared with the control group $(0 \mu \mathrm{MRb} 1)$, ${ }^{* *} p<0.001$, compared with the control group $(0 \mu \mathrm{M} R \mathrm{Rb})$

with the in-vivo results described above (Fig. 3a). The protein levels of both pPPAR $\gamma$ and PPAR $\gamma$ were increased in the Rb1 group (Fig. 5g-h). There was no significant difference in the pPPAR $\gamma /$ PPAR $\gamma$ ratio among the groups (Fig. 5i). Taken together, these results indicated that Rb1 attenuated lipid storage, promoted lipid excretion and influenced adipokine levels in adipocytes through the PPAR $\gamma$-AQP7 pathway.

\section{Discussion}

In this study, we found that $\mathrm{Rb} 1$ treatment reduced body weight, diminished fat mass and adipocyte enlargement and improved glycolipid metabolism in HFD-induced obese C57BL/6 mice. In differentiated 3 T3-L1 adipocytes, $\mathrm{Rb} 1$ decreased lipid droplet size. Rb1 augmented the expression levels of AQP7, influencing lipid metabolism both at both the animal and cellular levels. Rb1-mediated upregulation of the AQP7 protein was dependent on the PPAR $\gamma$ signaling pathway. A PPAR $\gamma$ inhibitor partially abrogated the beneficial effects of Rb1 on glycolipid metabolism. These results indicated that $\mathrm{Rb} 1$ promoted AQP7 expression via the PPAR $\gamma$ signaling pathway to ameliorate obesity status both in vivo and in vitro.

Obesity is closely associated with energy-balance dysregulation under the influence of environmental and genetic factors $[2,26]$. The anti-obesity function of $\mathrm{Rb} 1$ might be related to energy balance. A study reported that administration of Rb1 significantly suppressed food intake and increased energy expenditure in HFDinduced obese mice [27]. Adipocyte hypertrophy and excessive adipose accumulation are biological characteristics of obesity. The principal metabolic comorbidity 


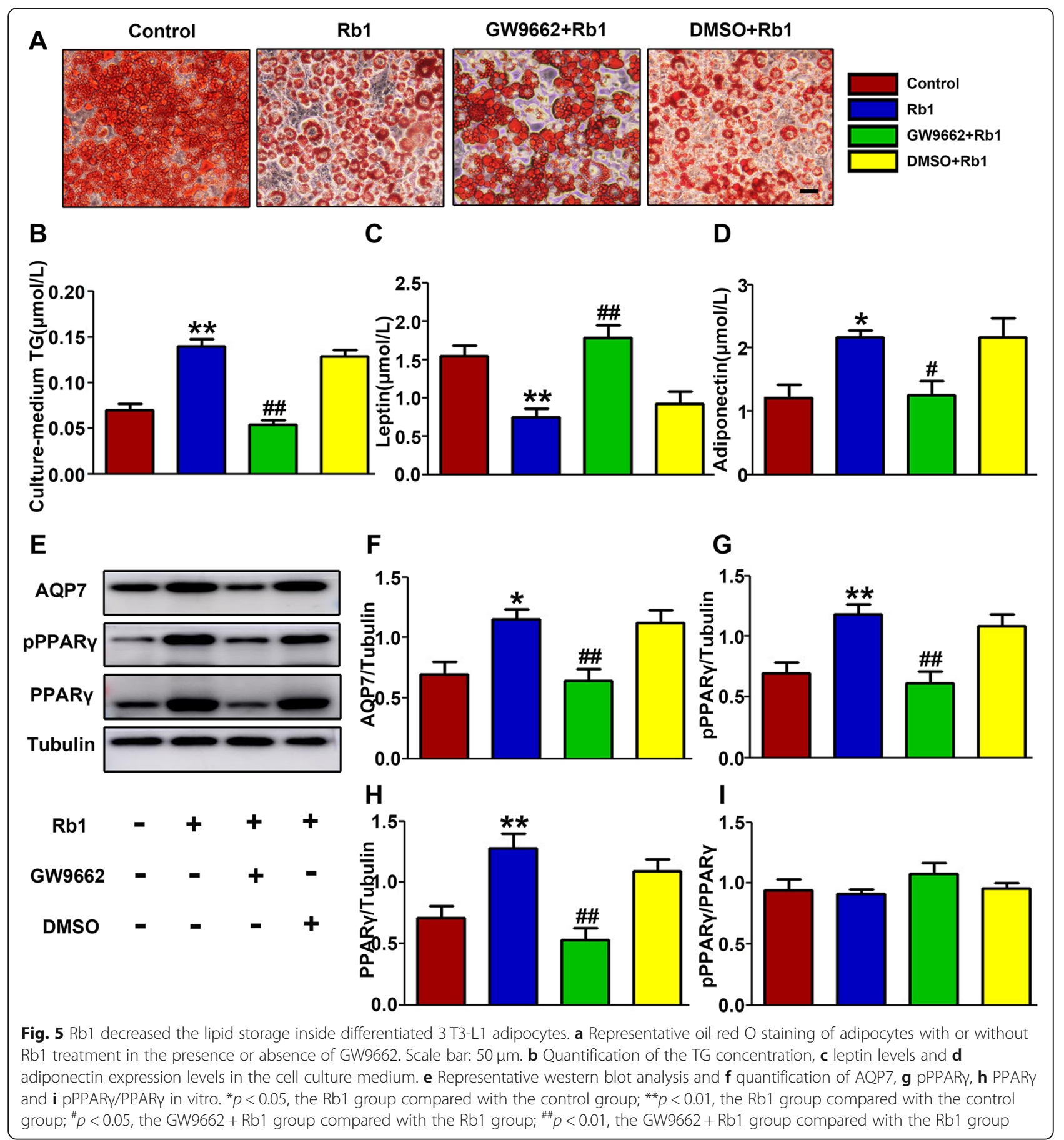

associated with excessive body fat is dyslipidemia [28]. Lipogenesis and lipolysis in adipocytes are essential processes for the maintenance of lipid homeostasis. Adipocytes, the main component of adipose tissues, continuously synthesize and hydrolyze triglycerides in response to energy balance.

Ginseng, the root of Panax ginseng, is believed to delay senility and promote longevity. Ginsenosides, the most biologically active components of ginseng, exert multiple beneficial effects in the circulatory, endocrine, immune and central nervous systems [29]. Rb1 is the most abundant and representative ginsenoside. We first demonstrated a new role for Rb1 against obesity. Our study shows that Rb1 significantly ameliorated glycolipid metabolism and diminished body weight without damaging liver function (Supplement 2). We have proven that Rb1 could significantly diminish the body weights of HFDinduced obese mice. According to previous studies, the 
possible molecular mechanisms of its anti-obesity effects may involve the phosphatidylinositol 3-kinase (PI3K)/ Akt signaling pathway and neuropeptide $\mathrm{Y}$ in the central nervous system $[13,27]$.

Regulation of AQP7 in adipose tissue appears crucial for obesity treatment. Human and rodent adipose tissue expresses AQP7 mRNA, but not that of other AQPs, in high abundance. AQP7 is involved in the process of adipogenesis and the transport of triglycerides in adipocytes [30]. The downregulation of AQP7 expression is closely related to the occurrence of type 2 diabetes and obesity [31]. However, AQP7 expression varies in the different adipose depots of obese people [32]. A sex-related difference in the effects of exercise training on AQP7 were also observed, which might be correlated with estradiol [33]. Evidence regarding the relationship between AQP7 expression and type 2 diabetes is still lacking. In this study, we found that Rb1 increased AQP7 expression both in vitro and in vivo. Body weight and fat mass in obese mice and lipid accumulation in mature 3 T3-L1 adipocytes were decreased under $\mathrm{Rb} 1$ treatment. Increased AQP7 expression and improved glycometabolism were observed in obese mice. With the increase in AQP7, lipid storage inside adipocytes was significantly decreased by Rb1. In addition, serum TG levels tended to be decreased in obese mice with Rb1 treatment.

To date, the most common reported regulators of AQP7 are insulin, leptin, and PPAR $[12,34]$. With the suppression of $A Q P 7$, plasma leptin concentrations were shown to be positively correlated with body fat mass [35]. We revealed that Rb1 decreased the leptin concentration in cell culture medium of adipocytes, but whether this phenomenon is correlated with the change in AQP7 expression is still unknown. As a master gene of adipogenesis, PPAR $\gamma$ can upregulate the expression of AQP7. PPAR $\gamma$, which is mainly expressed in adipose tissue and the immune system, is closely related to the differentiation of adipocytes, glycolipid metabolism and insulin resistance [36]. Thiazolidinediones, insulin sensitizers that stimulate PPAR $\gamma$, increased AQP7 mRNA abundance in the adipose tissue of male rodents [37]. Our study demonstrated that $\mathrm{Rb} 1$ can regulate AQP7 and increase glycerol release from adipocytes by activating PPARy. All effects of Rb1 on adipose tissue and adipocytes determined in this experiment could be partially reversed by GW9662, an inhibitor of PPAR $\gamma$.

In addition to its genetic regulation, PPAR $\gamma$ undergoes a variety of posttranscriptional modifications. Both of these mechanisms determine its specific activities [38]. The posttranscriptional modifications of PPARY include serine phosphorylation, acetylation and lysine sumoylation [39]. Phosphorylation of PPAR $\gamma$ at Ser273 is essential for insulin resistance related to obesity [40, 41]. Dephosphorylation of Ser112 is associated with adipogenesis, and the phosphorylation of PPAR at Ser112 strongly inhibits adipogenesis [42, 43]. Our study showed that $\mathrm{Rb} 1$ could promote the expression of pPPAR (Ser112), but no significant increase in PPPAR (Ser112) / PPAR $\gamma$ was observed. This finding suggested that $\mathrm{Rb} 1$ plays an important role in the upregulation of PPAR $\gamma$ rather than the phosphorylation of PPAR $\gamma$.

Considering the pathophysiological feature of obesity, turnover of FFAs and glycerol is vital for glycolipid metabolism. Adipocyte glycerol permeability is a key element in the regulation of fat accumulation. An increase in FFAs can sensitively reflect dyslipidemia. Obese individuals produce excessive FFAs, which can accelerate the development of metabolic syndrome and cardiovascular disease. Wang et al. found that $\mathrm{Rb} 1$ could reduce oxidative stress and inflammation in $3 \mathrm{~T} 3-\mathrm{L} 1$ adipocytes by reducing the FFA content [44]. Shang et al. found that $\mathrm{Rb} 1$ could improve heterotopic lipid deposition and downregulate FFA levels in obese mice [45]. In this study, $\mathrm{Rb} 1$ treatment significantly reduced lipid accumulation and increased the secretion of TG in 3 T3-L1 adipocytes. In addition, Rb1 significantly reduced the plasma level of FFAs and the size of the adipocytes. These results showed that $\mathrm{Rb} 1$ has potential value in promoting the transport of lipids in adipocytes. However, there were no significant differences in the plasma levels of TC, HDL-C, LDL-C, TGs or blood sugar between the control group and the Rb1 group. This might be related to the complicated metabolic pathways in the lipids of different organs, as our experiment focused on only adipose tissue.

Intervention with $\mathrm{Rb} 1$ did not change serum lipid levels compared to those in the HFD group in this study, while triglyceride levels tended to be lower, but this difference was not significant. This finding seems inconsistent with previous studies [27]. Instead of ApoEknockout mice, we used wild-type C57BL/6 mice, the lipids of which are hard to regulate. In addition, a 5week intervention time maybe not long enough for Rb1 to significantly reverse lipid metabolism. In addition, drug delivery methods also influence intracellular concentrations in vivo.

\section{Conclusion}

Our results indicate that ginsenoside $\mathrm{Rb} 1$ can promote lipid transport and ameliorate obesity by upregulating AQP7 through the PPARY pathway. Our study suggests $\mathrm{Rb} 1$ as a promising antiobesity therapeutic through its targeting of the transportation of lipids.

\section{Supplementary information}

Supplementary information accompanies this paper at https://doi.org/10. 1186/s12986-020-00490-8.

Additional file 1: Supplement 1. Variations in the body weight of mice on different diets with or without Rb1 treatment. IP: intraperitoneal 
injection. ${ }^{* *} p<0.01$, the HFD group compared to the Chow group: ${ }^{*} p<$ 0.05, the HFD group compared to the HFD + Rb1 group.

Additional file 2: Supplement 2. Liver function in mice from different groups. (A) Quantification of ALT serum levels. (B) Quantification of AST serum levels. ${ }^{*} p<0.05$, the HFD + Rb1 group compared to the HFD group; $" p<0.05$, the HFD + GW9662 + Rb1 compared to the HFD + Rb1 group.

\section{Abbreviations}

Rb1: Ginsenoside Rb1; HFD: High-Fat Diet; FFA: Free Fatty Acid; PPARY: Peroxisome Proliferator-activated Receptor Gamma; AQP7: Aquaporin 7; AQPS: Aquaporins; PPRE: Peroxisome Proliferator-activated Receptor Response Element; DMSO: Dimethylsulfoxide; BFR: Body Fat Rate; IPGT T: Intraperitoneal Glucose Tolerance Test; DMEM: Dulbecco's Modified Eagle Medium; IBMX: 3-isobutyl-1-methylxanthine; pPPARy: Phosphorylated

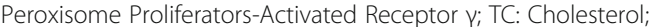
TG: Triglyceride; HDL-C: High Density Lipoprotein Cholesterol; LDL-C: Low Density Lipoprotein Cholesterol

\section{Acknowledgements}

\section{Not applicable.}

\section{Authors' contributions}

$R G, L W, X Q Z, M H L, P Z, H L L, M D$, and $H X L$ made substantial contributions to the conception. RG, LW and XQZ implemented experiments. MHL and PZ analyzed the data and made figures for this manuscript. RG, LW and MD wrote the original draft and substantively revised it. $\mathrm{HXL}$ administrated this project. HXL, MD and HLL funded this experiment. All authors read and approved the final manuscript.

\section{Funding}

This work was supported by the National Natural Science Foundation of China (81873516), the National Key Research and Development Program of China (2017YFC1308303), the Key Technology Research and Development Program of Shandong Province (2017GSF218045), the Joint Research Funds for Shandong University and Karolinska Institute (SDU-KI-2019-10), the natural science foundation of Fujian Province (2016 J01441) and Fujian provincial young and middle-aged backbone foundation (2016-ZQN-55).

\section{Availability of data and materials}

The datasets used or analyzed during the current study are available from the corresponding author on reasonable request.

\section{Ethics approval and consent to participate}

This study was approved by the ethics committee of Qilu Hospital, Cheeloo College of Medicine, Shandong University.

\section{Consent for publication}

Not applicable.

\section{Competing interests}

The authors declare that they have no competing interests.

\section{Author details}

${ }^{1}$ The Key Laboratory of Cardiovascular Remodeling and Function Research, Chinese Ministry of Education, Chinese National Health Commission and Chinese Academy of Medical Sciences, The State and Shandong Province Joint Key Laboratory of Translational Cardiovascular Medicine, Department of Cardiology, Qilu Hospital, Cheeloo College of Medicine, Shandong University, 107 Wenhuaxi Road, Jinan 250012, China. ²Department of Cardiology, The Second Affiliated Hospital of Fujian Medical University, Quanzhou 362000, Fujian, People's Republic of China. ${ }^{3}$ Department of Cardiology, Ji'an Municipal Center People's Hospital, Ji'an, Jiangxi, China. ${ }^{4}$ State Key Laboratory of Cardiovascular Disease, Fuwai Hospital, National Center for Cardiovascular Diseases, Chinese Academy of Medical Sciences and Peking Union Medical College, Beijing 100037, People's Republic of China.
Received: 23 January 2020 Accepted: 6 August 2020 Published online: 17 August 2020

\section{References}

1. Fruh SM. Obesity: risk factors, complications, and strategies for sustainable long-term weight management. J Am Assoc Nurse Pract. 2017;29(S1):S3S14.

2. Heymsfield SB, Wadden TA. Mechanisms, pathophysiology, and Management of Obesity. N Engl J Med. 2017;376(3):254-66.

3. Engin A. Fat cell and fatty acid turnover in obesity. Adv Exp Med Biol. 2017; 960:135-60.

4. Arner P, Bernard S, Salehpour M, Possnert G, Liebl J, Steier P, et al. Dynamics of human adipose lipid turnover in health and metabolic disease. Nature. 2011;478(7367):110-3.

5. Agre P. Nobel lecture. Aquaporin water channels. Biosci Rep. 2004;24(3): 127-63.

6. da Silva IV, Soveral G. Aquaporins in obesity. Adv Exp Med Biol. 2017;969: 227-38.

7. Madeira A, Moura TF, Soveral G. Aquaglyceroporins: implications in adipose biology and obesity. Cell Mol Life Sci. 2015;72(4):759-71.

8. Rojek A, Praetorius J, Frokiaer J, Nielsen S, Fenton RA. A current view of the mammalian aquaglyceroporins. Annu Rev Physiol. 2008;70:301-27.

9. Hara-Chikuma M, Sohara E, Rai T, Ikawa M, Okabe M, Sasaki S, et al. Progressive adipocyte hypertrophy in aquaporin-7-deficient mice: adipocyte glycerol permeability as a novel regulator of fat accumulation. J Biol Chem. 2005;280(16):15493-6.

10. Hibuse T, Maeda N, Funahashi T, Yamamoto K, Nagasawa A, Mizunoya W, et al. Aquaporin 7 deficiency is associated with development of obesity through activation of adipose glycerol kinase. Proc Natl Acad Sci U S A. 2005;102(31):10993-8.

11. Fruhbeck G, Catalan V, Gomez-Ambrosi J, Rodriguez A. Aquaporin-7 and glycerol permeability as novel obesity drug-target pathways. Trends Pharmacol Sci. 2006;27(7):345-7.

12. Kishida K, Shimomura I, Nishizawa H, Maeda N, Kuriyama H, Kondo H, et al Enhancement of the aquaporin adipose gene expression by a peroxisome proliferator-activated receptor gamma. J Biol Chem. 2001;276(51):48572-9.

13. Lin N, Cai DL, Jin D, Chen Y, Shi JJ. Ginseng panaxoside Rb1 reduces body weight in diet-induced obese mice. Cell Biochem Biophys. 2014;68(1):18994.

14. Shang W, Yang $Y$, Zhou L, Jiang B, Jin H, Chen M. Ginsenoside Rb1 stimulates glucose uptake through insulin-like signaling pathway in 3T3-L1 adipocytes. J Endocrinol. 2008;198(3):561-9.

15. Park S, Ahn IS, Kwon DY, Ko BS, Jun WK. Ginsenosides Rb1 and Rg1 suppress triglyceride accumulation in 3T3-L1 adipocytes and enhance betacell insulin secretion and viability in Min6 cells via PKA-dependent pathways. Biosci Biotechnol Biochem. 2008;72(11):2815-23.

16. Qiao L, Zhang X, Liu M, Liu X, Dong M, Cheng J, et al. Ginsenoside Rb1 enhances atherosclerotic plaque stability by improving autophagy and lipid metabolism in macrophage foam cells. Front Pharmacol. 2017:8:727.

17. Zhang X, Liu MH, Qiao L, Zhang XY, Liu XL, Dong M, et al. Ginsenoside Rb1 enhances atherosclerotic plaque stability by skewing macrophages to the M2 phenotype. J Cell Mol Med. 2018;22(1):409-16.

18. Zeng X, Guo R, Dong M, Zheng J, Lin H, Lu H. Contribution of TLR4 signaling in intermittent hypoxia-mediated atherosclerosis progression. J Transl Med. 2018;16(1):106.

19. Wu Y, Huang XF, Bell C, Yu Y. Ginsenoside Rb1 improves leptin sensitivity in the prefrontal cortex in obese mice. CNS Neurosci Ther. 2018;24(2):98-107.

20. Mu Q, Fang X, Li X, Zhao D, Mo F, Jiang G, et al. Ginsenoside Rb1 promotes browning through regulation of PPARgamma in 3T3-L1 adipocytes. Biochem Biophys Res Commun. 2015;466(3):530-5.

21. Chan LS, Yue PY, Kok TW, Keung MH, Mak NK, Wong RN. Ginsenoside-Rb1 promotes adipogenesis through regulation of PPARgamma and microRNA27b. Horm Metab Res. 2012:44(11):819-24.

22. Katano T, Ito Y, Ohta K, Yasujima T, Inoue K, Yuasa H. Functional characteristics of aquaporin 7 as a facilitative glycerol carrier. Drug Metab Pharmacokinet. 2014;29(3):244-8.

23. Kondo H, Shimomura I, Kishida K, Kuriyama H, Makino $Y$, Nishizawa H, et al. Human aquaporin adipose (AQPap) gene. Genomic structure, promoter analysis and functional mutation. Eur J Biochem. 2002;269(7):1814-26.

24. Galic S, Oakhill JS, Steinberg GR. Adipose tissue as an endocrine organ. Mol Cell Endocrinol. 2010;316(2):129-39. 
25. Yadav A, Kataria MA, Saini V, Yadav A. Role of leptin and adiponectin in insulin resistance. Clin Chim Acta. 2013;417:80-4.

26. Kopelman PG. Obesity as a medical problem. Nature. 2000;404(6778):635-43.

27. Xiong $Y$, Shen $L$, Liu K, Tso P, Xiong Y, Wang G, et al. Antiobesity and antihyperglycemic effects of ginsenoside Rb1 in rats. Diabetes. 2010;59(10): 2505-12.

28. Bays HE, Toth PP, Kris-Etherton PM, Abate N, Aronne LJ, Brown WW, et al. Obesity, adiposity, and dyslipidemia: a consensus statement from the National Lipid Association. J Clin Lipidol. 2013;7(4):304-83.

29. Kim JH. Pharmacological and medical applications of Panax ginseng and ginsenosides: a review for use in cardiovascular diseases. J Ginseng Res. 2018;42(3):264-9.

30. Hibuse T, Maeda N, Nagasawa A, Funahashi T. Aquaporins and glycerol metabolism. Biochim Biophys Acta. 2006;1758(8):1004-11.

31. Prudente S, Flex E, Morini E, Turchi F, Capponi D, De Cosmo S, et al. A functional variant of the adipocyte glycerol channel aquaporin 7 gene is associated with obesity and related metabolic abnormalities. Diabetes. 2007; 56(5):1468-74

32. Miranda M, Escote X, Ceperuelo-Mallafre V, Alcaide MJ, Simon I, Vilarrasa N, et al. Paired subcutaneous and visceral adipose tissue aquaporin-7 expression in human obesity and type 2 diabetes: differences and similarities between depots. J Clin Endocrinol Metab. 2010;95(7):3470-9.

33. Jin B, Chen X, Xing L, Xu W, Fu X, Zhu J, et al. Tissue-specific effects of estrogen on glycerol channel aquaporin 7 expression in an ovariectomized mouse model of menopause. Climacteric. 2017;20(4):385-90.

34. Rodriquez A, Catalan V, Gomez-Ambrosi J, Garcia-Navarro S, Rotellar F, Valenti $V$, et al. Insulin- and leptin-mediated control of aquaglyceroporins in human adipocytes and hepatocytes is mediated via the PI3K/Akt/mTOR signaling cascade. J Clin Endocrinol Metab. 2011;96(4):E586-97.

35. Maffei M, Halaas J, Ravussin E, Pratley RE, Lee GH, Zhang Y, et al. Leptin levels in human and rodent: measurement of plasma leptin and Ob RNA in obese and weight-reduced subjects. Nat Med. 1995;1(11):1155-61.

36. Kawai T, Masaki T, Doi S, Arakawa T, Yokoyama Y, Doi T, et al. PPAR-gamma agonist attenuates renal interstitial fibrosis and inflammation through reduction of TGF-beta. Lab Investig. 2009:89(1):47-58.

37. Lee DH, Park DB, Lee YK, An CS, Oh YS, Kang JS, et al. The effects of thiazolidinedione treatment on the regulations of aquaglyceroporins and glycerol kinase in OLETF rats. Metabolism. 2005;54(10):1282-9.

38. Janani C, Ranjitha Kumari BD. PPAR gamma gene--a review. Diabetes Metab Syndr. 2015;9(1):46-50.

39. Ahmadian M, Suh JM, Hah N, Liddle C, Atkins AR, Downes M, et al. PPARgamma signaling and metabolism: the good, the bad and the future. Nat Med. 2013;19(5):557-66.

40. Choi JH, Banks AS, Kamenecka TM, Busby SA, Chalmers MJ, Kumar N, et al. Antidiabetic actions of a non-agonist PPARgamma ligand blocking Cdk5mediated phosphorylation. Nature. 2011;477(7365):477-81.

41. Choi JH, Banks AS, Estall JL, Kajimura S, Bostrom P, Laznik D, et al. Antidiabetic drugs inhibit obesity-linked phosphorylation of PPARgamma by Cdk5. Nature. 2010;466(7305):451-6.

42. Hu E, Kim JB, Sarraf $P$, Spiegelman BM. Inhibition of adipogenesis through MAP kinase-mediated phosphorylation of PPARgamma. Science. 1996; 274(5295):2100-3.

43. Li D, Zhang L, Xu L, Liu L, He Y, Zhang Y, et al. WIP1 phosphatase is a critical regulator of adipogenesis through dephosphorylating PPARgamma serine 112. Cell Mol Life Sci. 2017;74(11):2067-79.

44. Wang M, Chen Y, Xiong Z, Yu S, Zhou B, Ling Y, et al. Ginsenoside Rb1 inhibits free fatty acidsinduced oxidative stress and inflammation in 3T3L1 adipocytes. Mol Med Rep. 2017;16(6):9165-72.

45. Shang WB, Yu XZ, Wang GQ, Zhao J. Effect of ginsenoside Rb1 in ameliorating insulin resistance and ectopic fat deposition in obese mice induced by high fat diet. Zhongguo Zhong Yao Za Zhi. 2013;38(23):4119-23.

\section{Publisher's Note}

Springer Nature remains neutral with regard to jurisdictional claims in published maps and institutional affiliations.

\section{Ready to submit your research? Choose BMC and benefit from:}

- fast, convenient online submission

- thorough peer review by experienced researchers in your field

- rapid publication on acceptance

- support for research data, including large and complex data types

- gold Open Access which fosters wider collaboration and increased citations

- maximum visibility for your research: over $100 \mathrm{M}$ website views per year

At BMC, research is always in progress.

Learn more biomedcentral.com/submissions 